\title{
Circulating Microparticles from Patients with Obstructive Sleep Apnea Enhance Vascular Contraction
}

\section{Mandatory Role of the Endothelium}

\author{
Simon Tual-Chalot, ${ }^{* \dagger}$ Keita Fatoumata, ${ }^{* \dagger}$ \\ Pascaline Priou, ${ }^{\star \dagger \ddagger}$ Wojciech Trzepizur, ${ }^{\star \star \ddagger}$ \\ Abderahim Gaceb, ${ }^{* \dagger}$ Cristina Contreras, ${ }^{\S}$ \\ Dolores Prieto, ${ }^{\S}$ Maria Carmen Martinez, ${ }^{* \dagger}$ \\ Frédéric Gagnadoux, ${ }^{* \dagger \ddagger}$ and \\ Ramaroson Andriantsitohaina* ${ }^{\star \dagger}$
}

MP-treated mice. These data provide evidence that circulating MPs from patients with OSA induce ex vivo vascular hyperreactivity with the obligatory role of the endothelium and subtle interactions between the nitric oxide and cyclooxygenase pathways and metabolites. These results highlight the participation of MPs in vascular dysfunction associated with OSA. (Am J Pathol 2012, 181: 1473-1482; bttp://dx.doi.org/10.1016/j.ajpath.2012.06.020)

Obstructive sleep apnea (OSA) is a highly prevalent disease characterized by recurrent episodes of partial or complete obstruction of the upper airways during sleep, leading to repeated falls in oxygen saturation. OSA is associated with hypertension, coronary artery disease, stroke, and premature death, and considerable evidence is now available in support of an independent association between OSA and cardiovascular diseases. ${ }^{1}$ Various pathophysiologic mechanisms have been proposed to contribute to the pathogenesis of vascular dysfunction in patients with OSA. ${ }^{2}$ In this view, circulating microparticles (MPs) have been considered as biomarkers of vascular injury and inflammation in several cardiovascular abnormalities. ${ }^{3-5}$ Indeed, altered levels of MPs have been detected and frequently correlate with the severity of the pathologic condition. MPs are vesicles with procoagulant and proinflammatory properties shed from the plasma membrane of various cell types, such as circulating cells and cells from the vascular wall, during activation or apoptosis. ${ }^{6,7}$ Recently, MPs have also been considered

Supported by the INSERM, Université d'Angers, Hospital of Angers, Société de Pneumologie de Langue Française (F.G.), and Association Ligérienne pour le Traitement à Domicile, I'Innovation et la Recherche. S.T.-C. is the recipient of a doctoral fellowship from the French Education Ministry.

Accepted for publication June 26, 2012

Address reprint requests to Ramaroson Andriantsitohaina, Ph.D. INSERM U1063, Institut de Biologie en Santé, Rue des Capucins, Angers, F-49045 France. E-mail: ramaroson.andriantsitohaina@univ-angers.fr. duced by MPs from patients with OSA but had no effect on contraction in vessels from control and non-OSA 
as effectors to vehicle biological messages on the target cells. However, few studies set out to demonstrate the importance of MPs in OSA, and they mainly evoke an alteration of the origin of MP levels. ${ }^{8-11}$ We provided evidence that patients with OSA display the same levels of circulating MPs as healthy individuals but that levels of MPs from activated leukocytes $\left(\mathrm{CD} 6 \mathrm{~L}^{+} \mathrm{MPs}\right)$ are higher in patients with OSA. ${ }^{12}$ There is a positive correlation between circulating levels of $\mathrm{CD}_{2} \mathrm{~L}^{+} \mathrm{MPs}$ and nocturnal desaturations severity. ${ }^{12,13}$ The evening-to-morning change in activated leukocyte-derived MPs in patients with OSA provided evidence in support of a direct link between sleep-disordered breathing and nocturnal MP release. ${ }^{13}$ In vitro and ex vivo studies have also demonstrated that MPs from patients with OSA can induce endothelial dysfunction, reduce endothelial nitric oxide (NO) release, and increase endothelial adhesion molecule expression. ${ }^{12}$ These recent data suggest that MPs could play a role in the pathogenesis of endothelial dysfunction that may initiate the atherogenic process in OSA. However, to our knowledge, the role played by MPs in the regulation of vascular contractility regarding exaggerated vasoconstriction that occurs in patients with OSA ${ }^{14}$ has never been assessed. Therefore, the present study was designed to investigate the effects of MPs from patients with OSA on vascular reactivity in response to vasoconstrictor agonists. The mechanisms by which OSA MPs affect vascular contractility were also investigated. MPs were injected i.v. into mice to test their pathophysiologic relevance in vivo.

\section{Materials and Methods}

\section{Patients}

Consecutive male patients (19 to 70 years old) investigated by polysomnography or overnight respiratory recording in the Sleep Unit of the Department of Respiratory Medicine of Angers University, Angers, France, for suspected OSA were screened for the study. Exclusion criteria were previous treatment for OSA, body mass index (calculated as weight in kilograms divided by height in meters squared) $\geq 35$, history of coronary artery disease, heart failure, stroke, hypertension, diabetes mellitus, dyslipidemia, and treatment with any drug known to affect endothelial function. Patients with an apnea-hypopnea index (AHI) of $\geq 5$ events per hour were included in the OSA group. Patients with an $\mathrm{AHI}<5$ were included in the non-OSA (N-OSA) group. All the patients underwent evaluation of clinical profile and daytime sleepiness using the Epworth Sleepiness Scale. ${ }^{15}$ Standard in-laboratory overnight polysomnography was performed as previously described ${ }^{16}$ using a computerized recording system (CID 102; Cidelec, Angers, France) with the following channels: electroencephalogram, electrooculogram, chin electromyogram, arterial oxygen saturation (finger oximetry), nasal-oral airflow (pressure cannula), tracheal sound (suprasternal microphone), electrocardiogram, chest and abdominal wall motion (piezo electrodes), bilateral tibialis electromyogram, and body position. Overnight respiratory recording (CID 102 L; Cidelec) was performed as previously de- scribed ${ }^{17}$ with the same channels except for electroencephalogram, electrooculogram, chin electromyogram, and tibialis electromyogram. Respiratory events were scored manually using recommended criteria. ${ }^{18}$ Hypopneas had to be associated with $\geq 4 \%$ oxygen desaturation. The University of Angers ethics committee approved the study, and patients gave their informed consent.

\section{MP Isolation and Characterization}

MP characterization was performed in the morning after sleep recording, at approximately 7 or 8 AM, before breakfast. Routine laboratory tests, including glucose, glycated hemoglobin, triglycerides, total cholesterol, high-density lipoprotein cholesterol, low-density lipoprotein cholesterol, and blood cell count, were also performed using a morning blood sample. For MP isolation, blood samples were collected in EDTA tubes (Vacutainer; Becton Dickinson, Le Pont de Claix, France) from a peripheral vein using a 21-gauge needle to minimize platelet activation and were processed for assay within 2 hours. Samples were centrifuged for 20 minutes at $270 \times g$, and plasma was then harvested and centrifuged for 20 minutes at $1500 \times g$ to obtain platelet-free plasma (PFP). Two hundred microliters of PFP was frozen and stored at $-80^{\circ} \mathrm{C}$ until use. As previously described, ${ }^{4,12}$ the remaining PFP was subjected to two series of centrifugation at 21,000 $\times g$ for 45 minutes to eliminate plasma and to pellet MPs for studies, and supernatant was replaced by $0.9 \% \mathrm{NaCl}$ saline solution. Finally, MP pellets were suspended in $150 \mu \mathrm{L}$ of $0.9 \%$ saline salt solution and were stored at $4^{\circ} \mathrm{C}$ until subsequent use.

MP subpopulations were discriminated into PFP according to the expression of membrane-specific antigens by flow cytometry. MPs derived from platelets, lymphocytes, and endothelial cells were identified using antiCD41, anti-CD45, and anti-CD146 antibodies, respectively. Anti-CD62L antibody was used to identify MPs derived from activated L-selectin ${ }^{+}$leukocytes. Irrelevant human IgG was used as an isotype-matched negative control for each sample. Five microliters of PFP was incubated with $5 \mu \mathrm{L}$ of specific antibody (Beckman Coulter, Villepinte, France), and after 45 minutes of incubation, samples were diluted in $300 \mu \mathrm{L}$ of $0.9 \% \mathrm{NaCl}$. Annexin V-FITC (BioVision Research Products, Mountain View, CA) binding was used to count phosphatidylserine-expressing MPs. To determine the MP concentration, equal volumes of sample and FlowCount beads were then added to calculate the MP concentration, and samples were analyzed using a $500 \mathrm{MPL}$ system flow cytometer (Beckman Coulter). Regions corresponding to MPs were identified in forward and side-angle light scatter intensity dot plot representation set at logarithmic gain, depending on their diameter $(0.1$ to $1.0 \mu \mathrm{m})$. Sample analysis was stopped after counting 10,000 events.

\section{Vascular Reactivity}

All the animal studies were performed using approved institutional protocols and conformed to the Guide for the Care and Use of Laboratory Animals published by the US National Institutes of Health. Male Swiss mice (8 to 10 
weeks old) were treated by i.v. injection of MPs into the tail vein at the circulating levels detected in the blood of each patient (OSA group: range, 1276 to 52,581 MPs per microliter of plasma; N-OSA group: range, 2887 to 32,703 MPs per microliter of plasma) or $0.9 \% \mathrm{NaCl}$ saline solution (CTL), as previously performed in other studies. ${ }^{4,12}$ Thus, several patients in the N-OSA group had circulating levels of MPs greater than several patients in the OSA group. In addition, for all the experiments, the OSA and $\mathrm{N}$-OSA groups were matched for age, body mass index, and biological data.

Aortic rings were isolated from mice treated with MPs from patients or healthy individuals during 24 hours. The mouse aorta was removed and carefully cleaned of adhering fat and connective tissue and then was cut into rings with or without endothelium (1.5 to $2 \mathrm{~mm}$ long) that were mounted on a wire myograph filled with physiologic saline solution as previously described. ${ }^{5,19}$ Concentration-response curves were constructed by cumulative application of serotonin $(5-\mathrm{HT}, 1 \mathrm{nmol} / \mathrm{L}$ to $10 \mu \mathrm{mol} / \mathrm{L}$; Sigma-Aldrich, St. Quentin, France) to vessels with functional endothelium in the absence or presence of the given inhibitor preincubated for 30 minutes: the NO synthase (NOS) inhibitor $N^{G}$-nitro-L-arginine (L-NA, 100 $\mu \mathrm{mol} / \mathrm{L}$; Sigma-Aldrich), the selective cyclooxygenase (COX)-2 inhibitor N-(2-cyclohexyloxy-4-nitrophenyl) methansulfonamide (NS-39810 $\mu \mathrm{mol} / \mathrm{L}$; Sigma-Aldrich), the nonselective COX inhibitor indomethacin $(100 \mu \mathrm{mol} / \mathrm{L}$; Sigma-Aldrich), the selective thromboxane $A_{2}$ receptor antagonist SQ-29548 (5 $\mu \mathrm{mol} / \mathrm{L} ;$ Sigma-Aldrich), L-NA plus indomethacin, the superoxide dismutase mimetic manganese (III) tetrakis-(1-methyl-4-pyridyl)-porphyrin pentachloride (MnTMPyP, $100 \mu \mathrm{mol} / \mathrm{L}$; Calbiochem, Darmstadt, Germany), or tetrahydrobiopterin (BH4, $10 \mu \mathrm{mol} / \mathrm{L}$; SigmaAldrich). All the inhibitors were used at the maximal active concentrations at which they inhibit the release of $\mathrm{NO}$ from all isoforms of NOSs, metabolites from COX-2 isoform, or metabolites from COX in blood vessels, as reported in many previous studies. ${ }^{4,20}$ Higher concentrations of L-NA, NS398, SQ-29548, or indomethacin did not induce further inhibition. Also, the contractile response to a cumulative concentration-response to the thromboxane $A_{2}$ agonist (9,11-dideoxy-11a,9a-epoxymethanoprostaglandin F2) (U46619; Cayman Chemicals, Ann Arbor, MI) or to the combination of U-46619 (100 nmol/L) and $\mathrm{KCl}(80 \mathrm{mmol} / \mathrm{L})$ was assessed in mouse aortas. Finally, a concentration-response curve to 5-HT was performed in aortas without functional endothelium. The endothelium was removed by rubbing the intimal surface of the rings with a rough needle. Aortas were considered denuded of their endothelium when acetylcholine $(1 \mu \mathrm{mol} / \mathrm{L})$ did not produce any relaxation.

\section{NO Determination by Electron Paramagnetic Resonance}

Detection of NO production was performed using a technique with $\mathrm{Fe}^{2+}$ diethyldithiocarbamate (Sigma-Aldrich) as spin trap as previously described. ${ }^{21}$ Briefly, 24 hours after administration of MPs, the animals were sacrificed.
The aortas were dissected and incubated for NO production in $250 \mu \mathrm{L}$ of Krebs-HEPES buffer solution, treated with $250 \mu \mathrm{L}$ of colloid $\mathrm{Fe}$ (diethyldithiocarbamate) ${ }_{2}$, and incubated for 45 minutes at $37^{\circ} \mathrm{C}$. NO detection was measured in situ by electron paramagnetic resonance, and values are expressed as amplitude signal per milligram tissue (dry weight).

\section{Western Blot Analysis}

Aortas were dissected, homogenized, and lysed. Proteins $(40 \mu \mathrm{g})$ were separated on NuPAGE gels (Invitrogen, Carlsbad, CA). Blots were probed with anti-endothelial NOS (eNOS) (BD Biosciences, San Jose, CA), anti-phospho-eNOS Thr 495, anti-phospho-eNOS Ser 1117 (Cell Signaling Technology Inc., Beverly, MA), and anti-COX-1 and 2 (Santa Cruz Biotechnology, Santa Cruz, CA) antibodies. A monoclonal mouse $\beta$-actin antibody (Sigma-Aldrich) was used at 1:1000 dilution to visualize protein gel loading. The membranes were then washed at least three times in Tris buffer solution containing $0.05 \%$ Tween and were incubated for 1 hour at room temperature with the appropriate horseradish peroxidase-conjugated secondary antibody. The protein antibody complexes were detected by ECL Plus reagent (Amersham Biosciences, Piscataway, NJ) according to the manufacturer's instructions.

\section{Determination of Prostanoid Production}

After sacrificing the mice pretreated with MPs, their aortas were dissected and treated with $5-\mathrm{HT}(1 \mu \mathrm{mol} / \mathrm{L}$, $37^{\circ} \mathrm{C}, 30$ minutes). After collection of the medium, thromboxane $\mathrm{A}_{2}$, prostaglandin $\mathrm{E}$ metabolite, prostacyclin, and total 8-isoprostane concentrations were measured by enzyme immunoassay kits (Cayman Chemicals). Prostanoid concentrations are expressed as pictograms per milliliter per milligram tissue (dry weight).

\section{Data Analysis}

Data were analyzed using GraphPad Prism software (GraphPad Software Inc., San Diego, CA). Data are expressed as mean \pm SEM, and $n$ represents the number of patients or experiments performed with mice. $\mathrm{pD}_{2}=$ $-\log \mathrm{EC}_{50}, \mathrm{EC}_{50}$ being the molar concentration of the agonist that produces $50 \%$ of the maximal effect; $E_{50}$ values were calculated by logit-log regression. For all the experiments, statistical analyses were performed using nonparametric U-tests or two-way analysis of variance for repeated measures and subsequent Bonferroni post hoc tests. $P<0.05$ was considered statistically significant.

\section{Results}

\section{Baseline Clinical and Biological Characteristics}

Thirty-four patients without any cardiovascular history of risk factor were included in this study. Patients were split into $\mathrm{N}$-OSA $(n=20)$ and OSA $(n=22)$ groups according to $\mathrm{AHI}$. 
Table 1. Characterization of the Study Population

\begin{tabular}{|c|c|c|c|}
\hline Characteristic & $\begin{array}{c}\text { N-OSA group } \\
(n=20)\end{array}$ & $\begin{array}{l}\text { OSA group } \\
(n=22)\end{array}$ & $P$ value \\
\hline Age (years) & $42.1 \pm 3$ & $53.8 \pm 1.9$ & 0.0143 \\
\hline Body mass index* & $25.8 \pm 1$ & $26.7 \pm 0.5$ & NS \\
\hline Weight (kg) & $82.4 \pm 4$ & $82.8 \pm 1.8$ & NS \\
\hline Systolic BP (mm Hg) & $124.1 \pm 4.1$ & $137.7 \pm 4.9$ & NS \\
\hline Diastolic BP $(\mathrm{mm} \mathrm{Hg})$ & $72.9 \pm 2$ & $80 \pm 3.1$ & NS \\
\hline Smokers (No.) & 5 & 3 & NS \\
\hline Plasma glucose (mmol/L) & $5.3 \pm 0.2$ & $5.7 \pm 0.3$ & NS \\
\hline Glycated hemoglobin (\%) & $5.7 \pm 0.1$ & $5.7 \pm 0.1$ & NS \\
\hline Total cholesterol (mmol/L) & $5.5 \pm 0.3$ & $5.8 \pm 0.2$ & NS \\
\hline Triglycerides $(\mathrm{g} / \mathrm{L})$ & $1.3 \pm 0.1$ & $1.5 \pm 0.2$ & NS \\
\hline HDL cholesterol (mmol/L) & $1.3 \pm 0.1$ & $1.3 \pm 0.1$ & NS \\
\hline LDL cholesterol (mmol/L) & $3.7 \pm 0.3$ & $3.9 \pm 0.2$ & NS \\
\hline $\begin{array}{l}\text { Epworth Sleepiness Scale } \\
\text { score }\end{array}$ & $9.3 \pm 1.5$ & $9.9 \pm 0.8$ & NS \\
\hline AHI (events/hour) & $1.3 \pm 0.3$ & $21.6 \pm 3.2$ & $<0.0001$ \\
\hline Mean $\mathrm{SaO}_{2}(\%)$ & $93.7 \pm 0.4$ & $93.2 \pm 0.3$ & NS \\
\hline $4 \%$ ODI (events/hour) & $1.5 \pm 0.5$ & $15.4 \pm 2$ & $<0.0001$ \\
\hline
\end{tabular}

All values are expressed as mean \pm SEM except where indicated otherwise.

${ }^{*}$ Calculated as weight in kilograms divided by height in meters squared.

$\mathrm{BP}$, blood pressure; HDL, high-density lipoprotein; LDL, low-density lipoprotein; ODI, oxygen desaturation index; NS, not significant; $\mathrm{SaO}_{2}$ oxyhemoglobin saturation.

The two groups differed by age but did not differ by weight, body mass index, blood pressure, daytime sleepiness as assessed by the Epworth Sleepiness Scale, ${ }^{15}$ lipid metabolism, glucose metabolism, or smoking rate (Table 1).

\section{Phenotype of Circulating MPs}

As previously described, ${ }^{12}$ the total number of circulating MPs was not significantly different between the N-OSA and OSA groups. Phenotypical characterization of MPs did not show significant differences between the N-OSA and OSA groups (Table 2). However, a positive correlation was found between $\mathrm{CD}_{2} \mathrm{~L}^{+} \mathrm{MPs}$ and the AHI (Figure 1).

Table 2. Circulating MP Levels

\begin{tabular}{|c|c|c|}
\hline MPs (events/ $\mu \mathrm{L}$ ) & $\begin{array}{l}\mathrm{N}-\text { OSA group } \\
\quad(n=20)\end{array}$ & $\begin{array}{l}\text { OSA group } \\
(n=22)\end{array}$ \\
\hline Total circulating MPs & $\begin{array}{l}13,125 \pm 2058 \\
(2887-32,703)\end{array}$ & $\begin{array}{l}20,957 \pm 3617 \\
(1276-52,581)\end{array}$ \\
\hline $\mathrm{CD} 41^{+} \mathrm{MPs}$ & $10,647 \pm 2016$ & $15,964 \pm 3059$ \\
\hline Annexin $\mathrm{V}^{+}$MPs & $4283 \pm 842$ & $5135 \pm 1913$ \\
\hline $\mathrm{CD} 45^{+} \mathrm{MPs}$ & $356 \pm 124$ & $244 \pm 79$ \\
\hline $\mathrm{CD} 6 \mathrm{~b}^{+} \mathrm{MPs}$ & $174 \pm 40$ & $145 \pm 21$ \\
\hline $\mathrm{CD} 2 \mathrm{~L}^{+} \mathrm{MPs}$ & $193 \pm 38$ & $260 \pm 42$ \\
\hline $\mathrm{CD} 2 \mathrm{P}^{+} \mathrm{MPs}$ & $257 \pm 48$ & $273 \pm 202$ \\
\hline $\mathrm{CD}^{146^{+}} \mathrm{MPs}$ & $306 \pm 64$ & $363 \pm 84$ \\
\hline $\mathrm{CD}_{235 \mathrm{a}^{+} \mathrm{MPs}}$ & $818 \pm 161$ & $502 \pm 71$ \\
\hline
\end{tabular}

Values are expressed as mean \pm SEM, and none are statistically significant. CD $41^{+}$MPs: platelet-derived MPs; Annexin $\mathrm{V}^{+}$MPs: procoagulant MPs; $C D 45^{+}$MPs: leukocyte-derived MPs; CD66b ${ }^{+}$MPs: granulocyte-derived MPs; $\mathrm{CD}_{2} \mathrm{~L}^{+} \mathrm{MPs}$ : L-selectin ${ }^{+} \mathrm{MPs}$; $\mathrm{CD} 6 \mathrm{P}^{+} \mathrm{MPs}$ : Pselectin+ MPs; CD146 ${ }^{+}$MPs: endothelial-derived MPs; CD235a ${ }^{+}$MPs: erythrocyte-derived MPs.

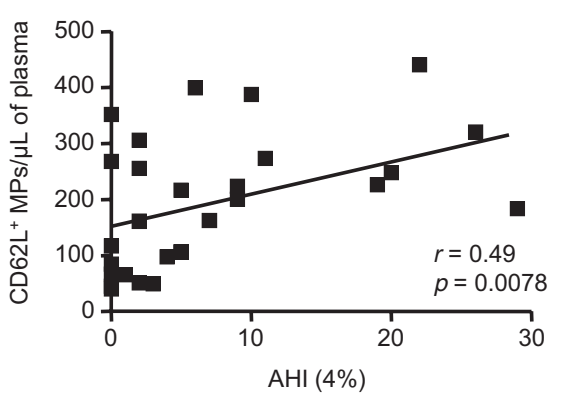

Figure 1. Positive correlation between $\mathrm{CD} 62 \mathrm{~L}^{+} \mathrm{MPs}$ and the severity of OSA according to the AHI.

\section{OSA MPs Increase Responsiveness of Mouse Aortic Rings to Vasoconstrictor Agents}

5-HT produced a concentration-dependent increase in tension in mouse aortic rings with functional endothelium in the CTL group and in those treated with circulating levels of either N-OSA or OSA MPs. Maximal response but not sensitivity to $5-\mathrm{HT}$ was markedly increased in mice treated with OSA MPs (mean \pm SEM maximal contractions, $3.1 \pm 0.2 \mathrm{mN} / \mathrm{mm}$ ) compared with those treated with either vehicle or $\mathrm{N}-\mathrm{OSA}$ MPs (mean \pm SEM maximal contractions, $2.3 \pm 0.2$ and $2.1 \pm 0.2 \mathrm{mN} / \mathrm{mm}$, respectively) (Figure 2A). Because concentrations of MPs used from the OSA and N-OSA groups were not significantly different and only OSA MPs induced vascular hyperreactivity, the effects observed cannot be related to the different concentrations of circulating MPs in both groups but rather to the composition of MPs.

As for $5-\mathrm{HT}$, aortic rings taken from mice treated with OSA MPs displayed increased maximal contraction in response to the stable analog of thromboxane $A_{2}$, $\mathrm{U}-46619$, compared with those from vehicle- and N-OSA MP-treated animals (Figure 2B). Finally, aortas from OSA MP-treated mice exhibited an increased contractile response to the concomitant application of $\mathrm{KCl}(100$ $\mathrm{mmol} / \mathrm{L}$ ) and a single concentration of U-46619 (100 $\mathrm{nmol} / \mathrm{L})$ compared with those from vehicle- and N-OSA MP-treated animals (Figure 2C).

Altogether, these data suggest that the OSA MP-induced vascular hyperreactivity is independent of the agonist or vasoconstrictor used. The OSA MP-induced vascular hyperreactivity may result from the release of vasoconstrictor products from different cellular origins (eg, endothelial cells, smooth muscle cells, or fibroblasts).

\section{Involvement of the NO Pathway in OSA MP-Induced Vascular Hyperreactivity}

To investigate the role of $\mathrm{NO}$, we studied the effect of the NOS inhibitor L-NA on the response to 5-HT. L-NA alone increased mean \pm SEM basal tone in aortas from control $(0.286 \pm 0.04 \mathrm{mN} / \mathrm{mm})$ and N-OSA MP-treated (0.576 \pm $0.14 \mathrm{mN} / \mathrm{mm}$ ) mice but not in those taken from mice treated with OSA MPs. Moreover, L-NA enhanced maximal response to 5-HT in control and N-OSA MP-treated vessels. In aortas from mice treated with OSA MPs, L-NA 

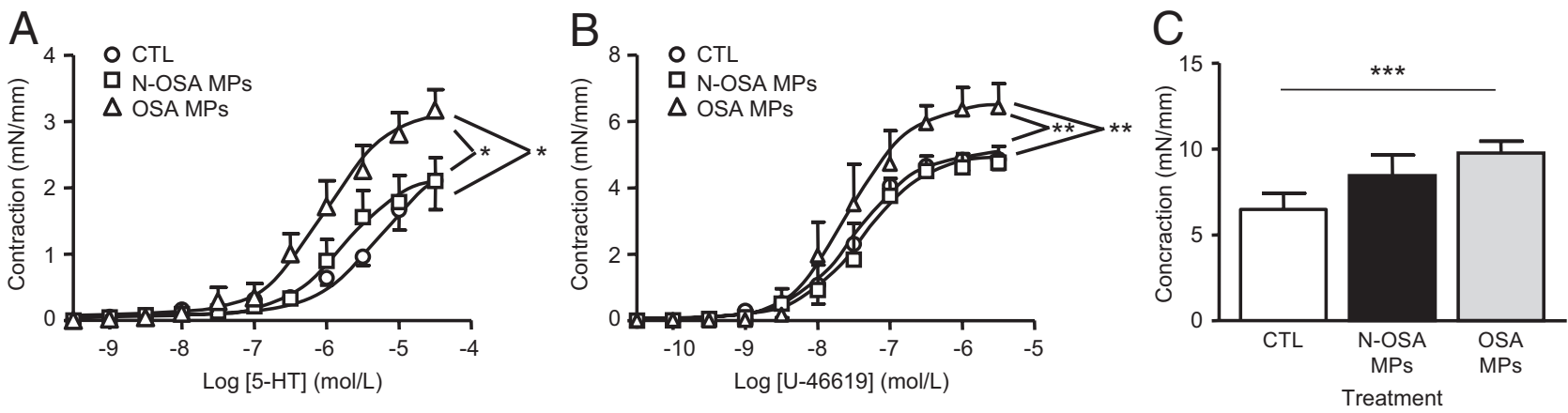

Figure 2. OSA MPs increase the responsiveness of mouse aortic rings to vasoconstrictor agents. Concentration-response curves to 5 -HT $(n=8)(\mathbf{A})$ and $\mathrm{U}-46619$ $(n=5)(\mathbf{B})$ in mouse aortic rings from vehicle- (CTL), N-OSA MP-, and OSA MP-treated mice. C: Contractile response to the concomitant application of KCl (80 $\mathrm{mmol} / \mathrm{L})$ and a single concentration of U-46619 $(1 \mu \mathrm{mol} / \mathrm{L})(n=6)$ in aortic rings from mice treated with vehicle, N-OSA MPs, or OSA MPs. Data are given as mean \pm SEM. ${ }^{*} P<0.001,{ }^{* *} P<0.01$, and ${ }^{* * * *} P<0.05$

did not affect maximal response to $5-\mathrm{HT}$. Moreover, it induced a rightward shift of the concentration-response curve to the agonist (mean $\pm \mathrm{SEM}$ pD being $5.7 \pm 0.2$ $5.5 \pm 0.3$, and $4.7 \pm 0.2$ for CTL, N-OSA, and OSA, respectively) (Figure 3 ). Thus, L-NA inhibited rather than potentiated the response to $5-\mathrm{HT}$ in aortas from mice treated with OSA MPs (Figure 3D). NO production in aortas taken from OSA MP-treated mice was significantly lower than that measured in vessels from vehicle- or N-OSA MP-treated mice (Figure 4A). To identify the molecular mechanism implicated in the decrease in NO production, we analyzed the levels of expression and phosphorylation of aortic eNOS by Western blot analysis. As shown in Figure 4B, eNOS expression was not significantly modified by MP treatment, although a trend toward an increase in eNOS expression was observed after treatment with MPs. However, treatment with OSA MPs, but not N-OSA MPs, induced a significant decrease in eNOS phosphorylation on the activator (Ser1177) and
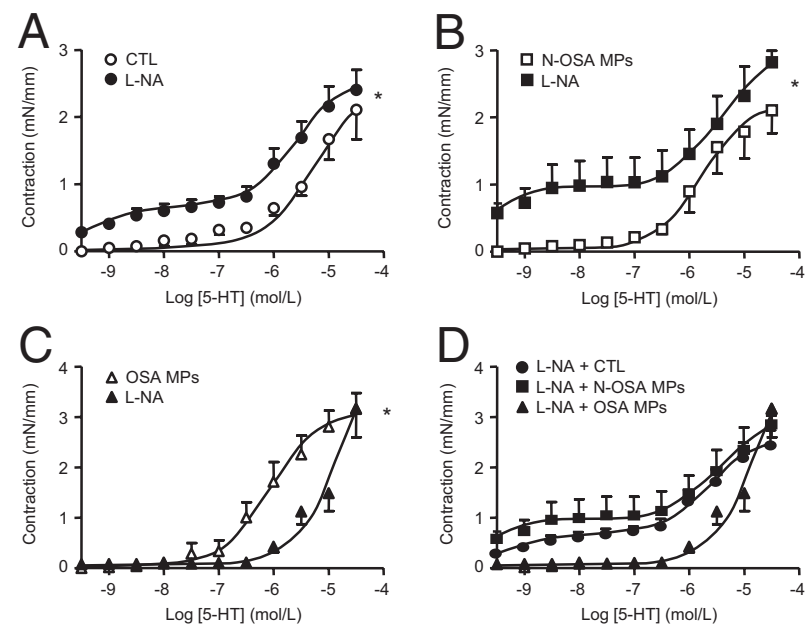

Figure 3. Involvement of the NO pathway in OSA MP-induced vascular hyperreactivity. Concentration-response curves to 5-HT in mouse aortic rings from vehicle-treated mice (CTL; $n=5)(\mathbf{A})$, N-OSA MP-treated mice $(n=5)$ (B), and OSA MP-treated mice $(n=5)(\mathbf{C})$ with or without the presence of NOS inhibitor (L-NA, $100 \mu \mathrm{mol} / \mathrm{L}$ ). D: Concentration-response curves to 5 -HT of aortic rings from mice treated with vehicle (CTL), N-OSA MPs-, or OSA-MPs in the presence of L-NA $(n=5)$. Data are given as mean \pm SEM. ${ }^{*} P<0.001$ inhibitor (Thr495) sites (Figure 4, C and D). After normalization of the amount of phosphorylated eNOS to the total amount of enzyme, the ratio of phosphorylated eNOS at the inhibitor and activation sites was greater in aortas from OSA MP-treated mice compared with those treated with N-OSA MPs or untreated (Figure 4E). These results indicate a down-regulation of eNOS activity caused by OSA MPs.

\section{A Superoxide Dismutase Mimetic-Sensitive Mechanism Is Involved in OSA MP-Induced Vascular Hyperreactivity}

Because superoxide anion production by uncoupled eNOS may account for the vascular hyperreactivity induced by OSA MPs, the effects of either MnTMPyP, a superoxide dismutase mimetic, or $\mathrm{BH} 4$ were analyzed in the contractile responses in aortas treated with MPs from patients in the N-OSA and OSA groups. Pretreatment with MnTMPyP of aortas from control or N-OSA MP-treated mice did not modify significantly the maximal contraction induced by $5-\mathrm{HT}$. The mean \pm SEM values of the maximal contraction were $2.3 \pm 0.2$ versus $2.05 \pm 0.1$ for vessels from control mice in the absence and presence of MnTMPyP, respectively. In vessels from N-OSA MP-treated mice, the mean \pm SEM values were $2.1 \pm 0.2$ versus 2.03 \pm 0.09 . In contrast, MnTMPyP induced an $\sim 33 \%$ reduction in the mean \pm SEM maximal contraction induced by $5-\mathrm{HT}$ of vessels from OSA MP-treated mice $(3.1 \pm 0.2$ versus $2.08 \pm 0.2$ in the absence and presence of MnTMPyP, respectively) without significant modification of the mean \pm SEM $\mathrm{pD}_{2}$ values $(6.04 \pm 0.02$ versus $5.9 \pm 0.10$ in the absence and presence of MnTMPyP, respectively). Furthermore, $\mathrm{BH} 4$ induced an increase in mean \pm SEM basal tone $(0.23 \pm 0.05 \mathrm{mN} / \mathrm{mm})$ but was unable to modify contraction to $5-\mathrm{HT}$ of aortas from mice treated with MPs from patients with OSA. Indeed, maximal contraction to $5-\mathrm{HT}$ in the presence of $\mathrm{BH} 4$ was $11 \%$ higher (not significant) than in the absence of $\mathrm{BH} 4$. Also, $\mathrm{pD}_{2}$ values were not statistically significantly different between both conditions, the mean \pm SEM values being $6.04 \pm 0.02$ versus $5.96 \pm 0.06$ in the absence and presence of $\mathrm{BH} 4$, respectively. 

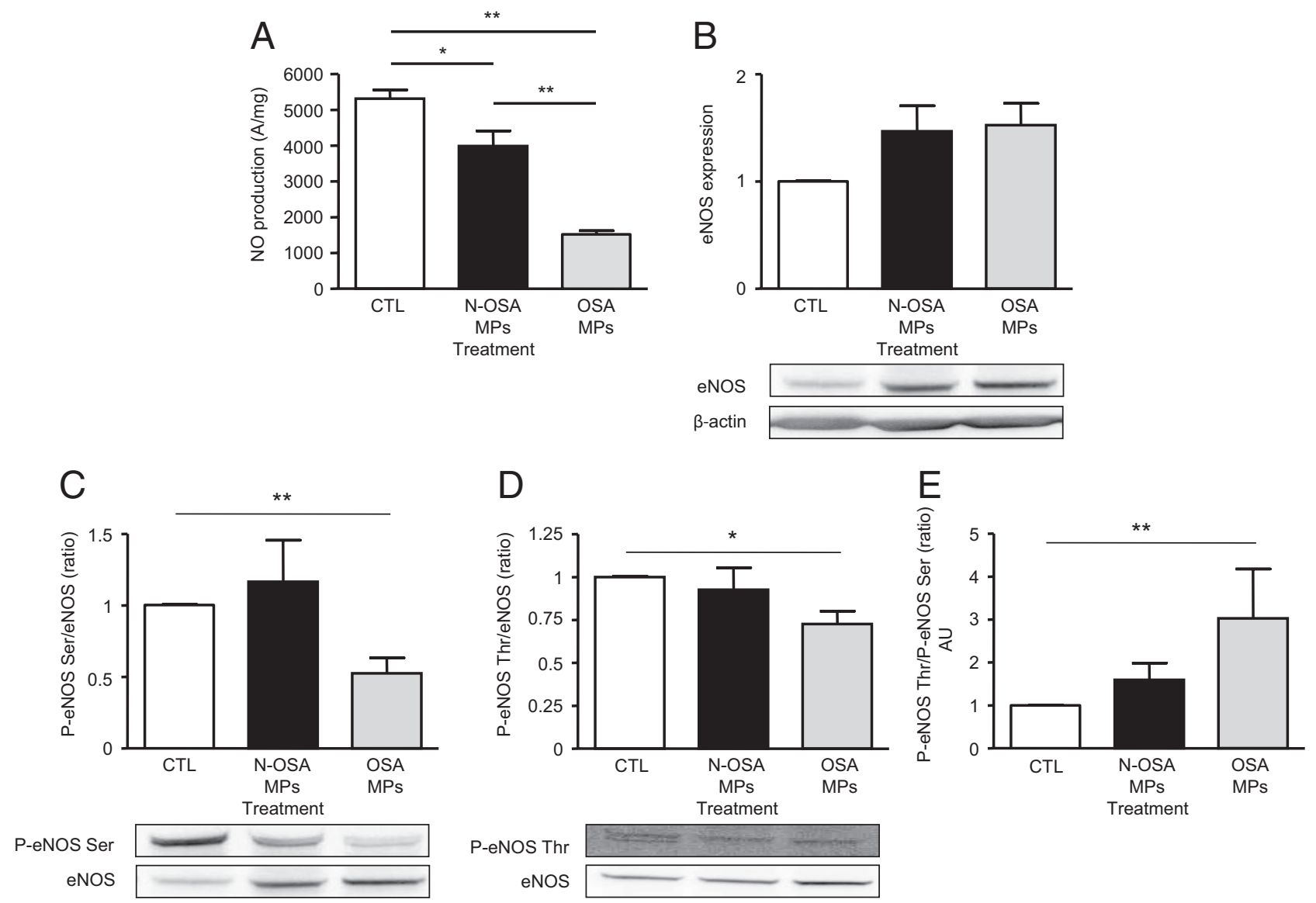

Figure 4. Down-regulation of eNOS activity by OSA MPs. A: Quantification of the amplitude of NO-Fe(diethyldithiocarbamate) ${ }_{2}$ signal in aortas from mice treated with vehicle (CTL), N-OSA MPs, and OSA MPs. Values are expressed as amplitude per milligram (A $/ \mathrm{mg})$ weight of dried aorta $(n=6)$. Western blots show eNOS expression (B), phospho-eNOS Ser 1177 (C), and phospho-eNOS Thr 495 (D), and histograms show the ratio of phophorylation of eNOS Ser 1177 (C) or eNOS Thr 495 (D) versus total eNOS. Immunoblots were quantified by densitometric analysis. Data are representative of five separate blots, and the densitometry values are expressed in arbitrary units (AU). E: The ratio of phosphorylation of eNOS at the inhibitory Thr 495) and activation (Ser 1177) sites is expressed in arbitrary units (AU). Data are given as mean \pm SEM. ${ }^{*} P<0.05,{ }^{* *} P<0.01$.

\section{Involvement of COX Metabolites in OSA MP-Induced Vascular Hyperreactivity}

To investigate the role of COX metabolites in 5-HT-induced vascular reactivity, the effects of a nonselective inhibitor of COX (indomethacin) and a selective inhibitor of COX-2 (NS-398) were examined. In the presence of indomethacin, the contractile response to $5-\mathrm{HT}$ was reduced in aortas from all groups of mice; the decrease was even greater in the OSA group (60\%) compared with the N-OSA and vehicle groups ( $47 \%$ and $48 \%$, respectively) (Table 3). When COX-2 was specifically silenced using NS-398, the response to 5-HT was impaired in vessels from controls, N-OSA MPs, and OSA MPs, with a stronger inhibition for aortas treated by OSA MPs (Table 3). These results suggest that OSA MP treatment leads to an increase in the release of vasoconstrictor metabolites derived from COX, including COX-2.

OSA MPs were able to enhance the expression levels of COX-1 and COX-2 compared with N-OSA MPs and vehicle (Figure $5, A$ and $B$ ). To test the hypothesis that COX activity could be affected by this treatment, we measured the production of different COX metabolites. Aortas treated with OSA-MPs displayed increased throm- boxane $\mathrm{A}_{2}$ and prostacyclin production compared with N-OSA MP-treated and untreated aortas. In contrast, release of prostaglandin $E_{2}$ or 8-isoprostane was not significantly different among the three groups (Figure 5, $\mathrm{C}-\mathrm{F})$. The hyperreactivity observed might be due to the increase in thromboxane $A_{2}$ production. To test this hypothesis, we performed a concentration-response curve to 5-HT in the presence of SQ-29548, a selective thromboxane $A_{2}$ receptor antagonist. SQ-29548 significantly reduced the response to $5-\mathrm{HT}$ in vessels from vehicleand N-OSA MP-treated mice but not in those from animals injected with OSA MPs (Table 3). These results suggest that the SQ-29548-sensitive pathway is involved in the response to 5-HT in vessels from vehicle- and $\mathrm{N}$-OSA MP-treated mice. In contrast, in those from OSA MP-treated mice, the SQ-29548-sensitive pathway might not be implicated in the response, or, alternatively, vasodilator metabolites might mask the effect of thromboxane $A_{2}$.

\section{Interaction between the NO and COX Pathways}

To study the eventual interaction between the NO and COX pathways, we used inhibitors, L-NA and indomethacin, in combination. On the one hand, blockade of the two pathways 
Table 3. $\mathrm{pD}_{2}$ Values and Maximal Contractions (Emax) of Serotonin Obtained in Aorta from Mice Treated with Vehicle (CTL), N-OSA MPs, or OSA MPs in the Absence or Presence of COX Inhibitor (Indomethacin, $10 \mu \mathrm{mol} / \mathrm{L}$ ), in the Presence of COX-2 Inhibitor (NS$398,1 \mu \mathrm{mol} / \mathrm{L}$ ), or in the Presence of Thromboxane $\mathrm{A}_{2}$ Antagonist (SQ-29548, $5 \mu \mathrm{mol} / \mathrm{L}$ )

\begin{tabular}{lrl}
\hline \multicolumn{1}{c}{ Treatment } & \multicolumn{1}{c}{$\mathrm{pD}_{2}$} & $\mathrm{Emax}(\mathrm{mN} / \mathrm{mm})$ \\
\hline CTL & & \\
Without inhibitors & $5.4 \pm 0.2$ & $2.3 \pm 0.2$ \\
With indomethacin & $6.3 \pm 0.2^{*}$ & $1.1 \pm 0.1^{*}$ \\
With NS-398 & $5.8 \pm 0.2$ & $1.3 \pm 0.1^{\star}$ \\
$\quad$ With SQ-29548 & $6 \pm 0.2$ & $1.2 \pm 0.1^{*}$ \\
N-OSA MPS & & \\
Without inhibitors & $5.8 \pm 0.2$ & $2.1 \pm 0.2$ \\
With indomethacin & $5.9 \pm 0.2$ & $1.1 \pm 0.1^{\star *}$ \\
With NS-398 & $5.5 \pm 0.2$ & $1.1 \pm 0.1^{\star * *}$ \\
With SQ-29548 & $5.9 \pm 0.1$ & $1.6 \pm 0.1^{*}$ \\
OSA MPS & & \\
Without inhibitors & $6.1 \pm 0.1$ & $3.1 \pm 0.2$ \\
With indomethacin & $5.6 \pm 0.3$ & $1.3 \pm 0.2^{\star *}$ \\
With NS-398 & $5.1 \pm 0.2$ & $2.2 \pm 0.4^{\star * *}$ \\
With SQ-29548 & $6 \pm 0.2$ & $3.3 \pm 0.3$ \\
\hline
\end{tabular}

All values are expressed as mean \pm SEM

${ }^{\star} P<0.05,{ }^{* *} P<0.001,{ }^{* \star *} P<0.01$ versus without inhibitors.

enhanced the response to 5-HT in aortas from control and $\mathrm{N}$-OSA MP-treated mice. On the other hand, the presence of the two blockers induced a rightward shift of the response to $5-\mathrm{HT}$ in vessels from mice treated with OSA MPs (Figure 6).

\section{Role of the Endothelium}

In vessels without functional endothelium, contraction to 5-HT was significantly reduced in vessels taken from mice treated with OSA MPs compared with those taken from control or N-OSA MP-treated mice (Figure 7). These results strongly suggest the obligatory role of the endothelium in the hyperreactivity of the aorta to 5-HT after treatment of mice with OSA MPs.

\section{Discussion}

In the present study, we showed that i.v. injection of OSA MPs into mice promotes vascular hyperreactivity in response to vasoconstrictor agents, with the obligatory role of the endothelium. This effect was associated with the ability of OSA MPs to reduce the activity of eNOS concomitant with a decrease in $\mathrm{NO}$ production. In addition, OSA MPs increased aortic COX-1 and COX-2 expression associated with enhanced production of thromboxane $A_{2}$ and prostacyclin, and indomethacin or the selective COX-2 inhibitor NS-398 reduced hyperreactivity in response to 5-HT. The SQ-29548-sensitive pathway might not be implicated in vascular hyperreactivity of OSA MPs, or, alternatively, vasodilator metabolites, including prostacyclin, might mask the effect of thromboxane $A_{2}$. Moreover, a mechanism sensitive to MnTMPyP is implicated in the vascular hyperreactivity induced by OSA MPs. These
A
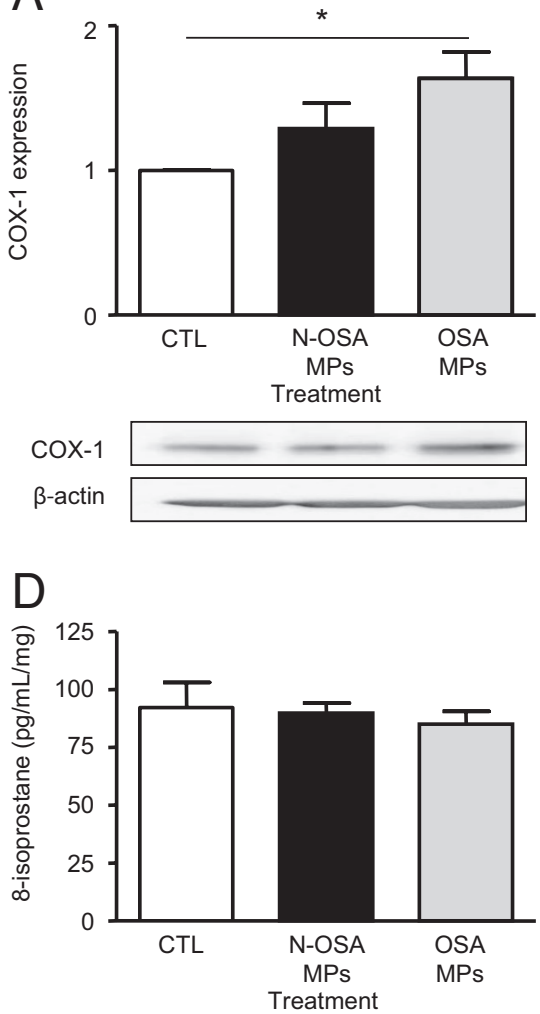

$\mathrm{B}$

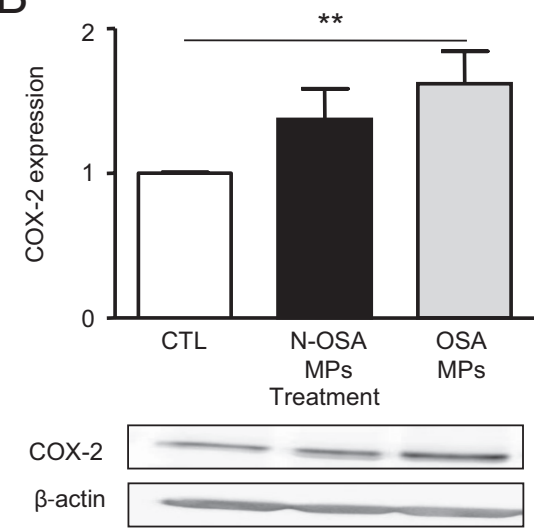

$\mathrm{E}$

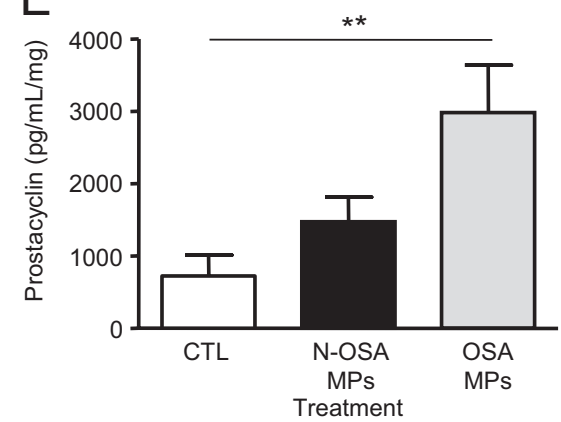

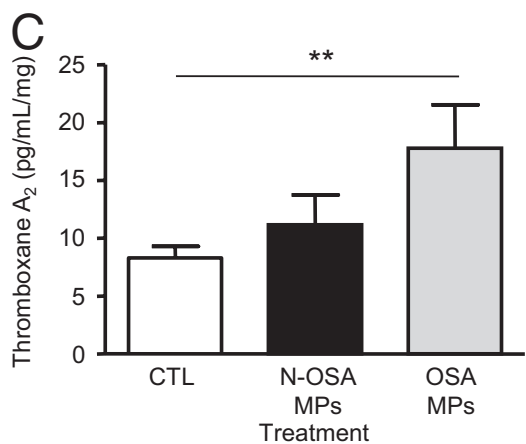

Treatment

Figure 5. OSA MPs and the COX pathway. Western blots reveal expression of COX-1 (A) and COX-2 (B) in aortas from mice treated with vehicle (CTL), N-OSA MPs, and OSA MPs. Data are representative of six separate blots. Concentrations of the COX derivatives thromboxane $\mathrm{A}_{2}(\mathbf{C})$, 8-isoprostane (D), prostacyclin (E), and prostaglandin $\mathrm{E}_{2}(\mathbf{F})$ in the supernatants of mouse aortas pretreated with vehicle (CTL), N-OSA MPs, and OSA MPs and stimulated with 5 -HT ( $n=5$ ). Data are given as mean \pm SEM. ${ }^{*} P<0.01,{ }^{* *} P<0.05$. 

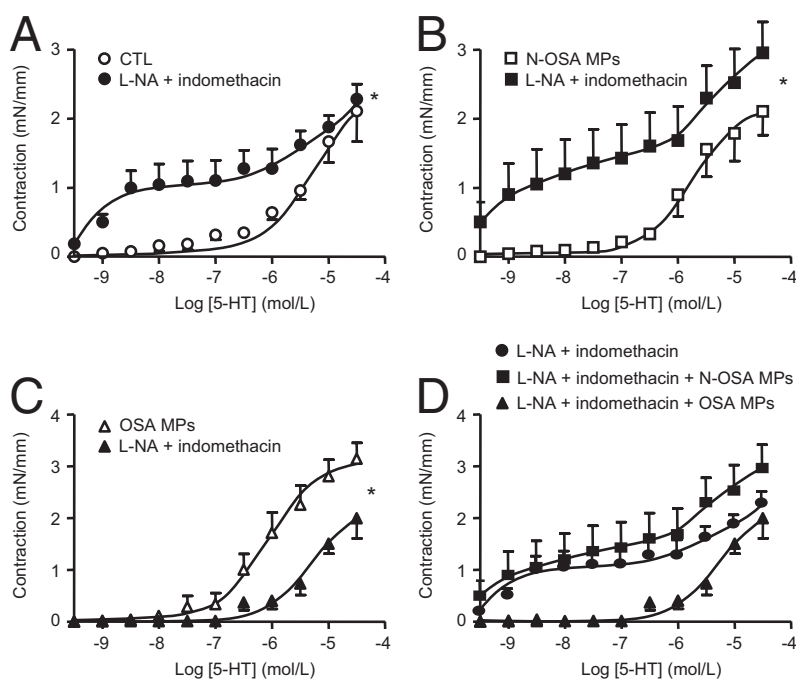

Figure 6. Interaction between the NO and COX pathways. Concentrationresponse curves to 5-HT in mouse aortic rings from vehicle-treated mice (CTL; $n=5)(\mathbf{A})$, N-OSA MP-treated mice $(n=5)(\mathbf{B})$, and OSA MP-treated mice $(n=$ 5) (C) in the absence or presence of L-NA plus indomethacin. D: Concentrationresponse curves to 5-HT of aortic rings from mice treated with vehicle (CTL), N-OSA MPs, or OSA MPs in the presence of L-NA plus indomethacin $(n=5)$ Data are given as mean \pm SEM. ${ }^{*} P<0.001$.

data provide evidence of the pathophysiologic relevance of MPs as vectors of transcellular exchange of messages in promoting endothelial ${ }^{12}$ and vascular (the present study) hyperreactivity in patients with OSA.

It is well accepted that MPs contribute, at least in part, to the alteration of vascular function in several cardiovascular diseases., ${ }^{6,7}$ Evidence of the pathophysiologic relevance of MPs in OSA was recently provided in vitro and ex vivo by their ability to impair endothelium-dependent relaxation in response to acetylcholine and to flow when injected into mice. ${ }^{12}$ In line with a previous study, ${ }^{12}$ circulating levels of MPs were not different between patients in the OSA and N-OSA groups, but levels of CD62 ${ }^{+}$ MPs were positively correlated with the severity of sleep-disordered breathing. We observed a significant difference in age between the N-OSA and OSA populations. Although we cannot exclude that age might affect the level of circulating MPs, it has been shown that MP levels from platelets, red blood cells, and those expressing phosphatidylserine in young patients are not different from those in elderly patients, ${ }^{22,23}$ suggesting that age does not affect MP generation or their procoagulant activity.

We provide further evidence that OSA MPs promote vascular hyperreactivity in response to vasoconstrictor agents in mouse aortas. These findings are in line with previous studies in which MPs from patients with septic shock enhance contraction in response to $5-\mathrm{HT} .{ }^{4}$ Septic MPs may, rather, be protective in counteracting the decrease in peripheral resistance and progressive hypotension during severe sepsis and explain the fact that increased levels of MPs may predict a more favorable outcome in severe sepsis in terms of mortality rate and organ dysfunction. ${ }^{24}$ In contrast to patients with septic shock, increased vascular reactivity in patients with OSA is deleterious and is probably involved in vascular re- modeling, leading to the exacerbation of vasoconstriction reported in this disease. Indeed, exaggerated vasoconstriction occurs in the forearms of patients with OSA in response to angiotensin $11 .{ }^{14}$ In a rat model of intermittent hypoxia, enhanced responses to endothelin-1 have also been reported in several vascular beds. ${ }^{25,26}$ Furthermore, increased contraction to norepinephrine in mouse hindquarters has also been observed. ${ }^{27}$ In contrast, contractile responses to potassium, endothelin-1, 5- $\mathrm{HT}$, and thromboxane $A_{2}$ mimetic were not affected in rat aortas after 35 days of intermittent hypoxia. ${ }^{26}$ Nevertheless, we provide evidence that MPs from OSA induced vascular hyperreactivity independently of the vasoconstrictor under the experimental conditions used and, therefore, participate in the regulation of vascular tone in this disease.

OSA MPs induced vascular hyperreactivity only in the presence of functional endothelium, suggesting the obligatory role of this tunica. In contrast, OSA MPs elicited vascular hyporeactivity in the absence of functional endothelium, suggesting that they can also act directly on smooth muscle cells. The pathways involved in this vascular hyporeactivity were not assessed since, in vivo, MPs interact with intact blood vessels in which the endothelium is present.

We previously reported decreased endothelial NO production in response to OSA MPs. ${ }^{12}$ These data corroborate studies showing a decreased plasma concentration of NO metabolites in OSA, which is correlated with OSA severity. ${ }^{28}$ In a previous work, ${ }^{12}$ MPs from patients with OSA reduced NO production from endothelial cells by negative regulation of eNOS activity. We reinforce this hypothesis inasmuch as i.v. injection of mice with OSA MPs decreased NO production in the aorta by a mechanism associated with reduced eNOS activity without alteration of its expression. Moreover, blockade of the NO pathway with L-NA alone increased basal tone in aortas from control and N-OSA MP-treated mice but not in those from mice treated with OSA MPs, suggesting that OSA MPs reduced basal NO production. Decrease of the basal level of $\mathrm{NO}$ has been reported in a rat model of intermittent hypoxia. ${ }^{29}$ Furthermore, L-NA enhanced maximal response to 5-HT in control and N-OSA MPtreated vessels. In aortas from mice treated with OSA

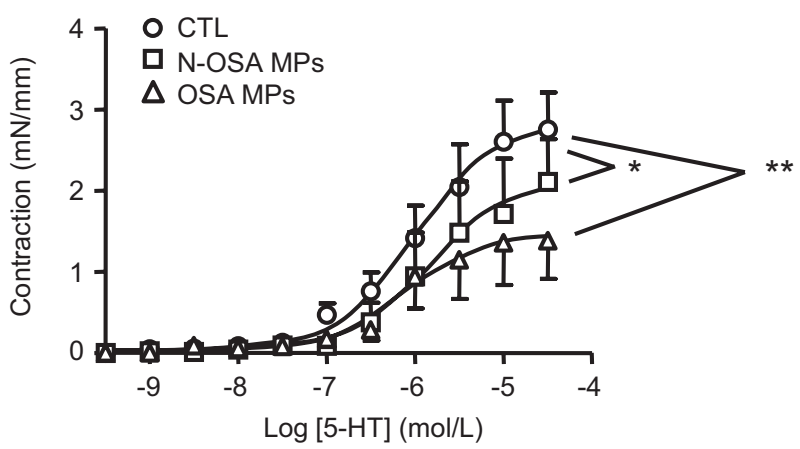

Figure 7. Role of the endothelium in the increased responsiveness to 5-HT induced by OSA MPs. Concentration-response curves to 5-HT without functional endothelium $(n=5)$ in mouse aortic rings from vehicle (CTL)-, N-OSA MP-, and OSA MP-treated mice. Data are given as mean \pm SEM. ${ }^{*} P<0.05$, ${ }_{* *} P<0.01$ 
MPs, L-NA did not affect maximal response to 5-HT. Altogether, these data demonstrate that decreased endothelial NO participates in vascular hyperreactivity elicited by OSA MPs. It is expected that the concentrationresponse curve to $5-\mathrm{HT}$ would remain unchanged in the presence of L-NA owing to reduced endothelial NO production in vessels from mice treated with OSA MPs. L-NA instead induced a rightward shift of the concentrationresponse curve to the agonist. One can advance the hypothesis that inhibition of NOS unmasks the existence of vasoconstrictor factor(s) released on OSA MP treatment. These factors might participate in the vascular hyperreactivity induced by OSA-MPs. Indeed, a plausible explanation may be the superoxide production via the uncoupled eNOS. Although the superoxide dismutase mimetic MnTMPyP decreased contraction to $5-\mathrm{HT}$ in vessels from mice treated with OSA MPs, incubation with $\mathrm{BH} 4$, an essential cofactor required for the activity of eNOS, had no effect. These results indicate that vascular hyperreactivity induced by OSA MPs may be due, at least in part, to increased reactive oxygen species production. The characterization of the source of reactive oxygen species remains to be evaluated.

The contraction in response to 5-HT involved the participation of COX vasoconstrictor metabolites in the aorta independent of the treatment (vehicle, N-OSA MPs, or OSA MPs). Blockade of either the nonselective COX inhibitor indomethacin or the COX-2 inhibitor resulted in a greater reduction in the 5-HT response in vessels from mice treated with OSA MPs, suggesting that COX metabolites participate, in part, in the increased response. Indeed, COX-1 and COX-2 expression was increased in aortas taken from OSA MP-treated mice. Up-regulation of COX-2 has been reported in endothelial cells treated with MPs from OSA ${ }^{12}$ and in venous endothelial cells harvested from patients with untreated OSA. ${ }^{30}$ The nature of the COX metabolites involved was found to be increased thromboxane $\mathrm{A}_{2}$ and prostacyclin. Concerning thromboxane $A_{2}$, blockade of thromboxane receptor by SQ-29548 significantly reduced the response to $5-\mathrm{HT}$ in vessels from vehicle- and N-OSA MP-treated mice but not in those from animals injected with OSA MPs. Thus, the SQ-29548-sensitive pathway is involved in the response to $5-\mathrm{HT}$ in vessels from vehicle- and N-OSA MP-treated mice. In contrast to OSA MP-treated mice, the SQ29548-sensitive pathway might not be implicated in the response, or, alternatively, vasodilator metabolites, probably prostacyclin, might mask the effect of thromboxane $A_{2}$. Moreover, another vasoconstrictor factor specific to COX metabolites might participate in OSA MP-induced vascular hyperreactivity. Indeed, concomitant blockade of the $\mathrm{NO}$ and COX pathways induced a rightward shift of the response to 5-HT in OSA MP-treated aortas. The nature of this vasoconstrictor factor remains to be identified.

In conclusion, we provide evidence that OSA MPs induce in vivo vascular hyperreactivity to vasoconstrictor agents in the aorta with the obligatory role of the endothelium, probably via increased production of reactive oxygen species. This effect is associated with a combination of reduced eNOS activity and NO production and a subtle interaction with the COX pathway and metabo- lites. The critical role of MPs as a vector of biological messages leading to vascular dysfunction in OSA is, therefore, underlined.

\section{References}

1. Bradley TD, Floras JS: Obstructive sleep apnoea and its cardiovascular consequences. Lancet 2009, 373:82-93

2. Shamsuzzaman AS, Gersh BJ, Somers VK: Obstructive sleep apnea implications for cardiac and vascular disease. JAMA 2003, 290: 1906-1914

3. Boulanger CM, Scoazec A, Ebrahimian T, Henry P, Mathieu E, Tedgui A, Mallat Z: Circulating microparticles from patients with myocardial infarction cause endothelial dysfunction. Circulation 2001, 104:26492652

4. Mostefai HA, Meziani F, Mastronardi ML, Agouni A, Heymes C, Sargentini C, Asfar P, Martinez MC, Andriantsitohaina R: Circulating microparticles from patients with septic shock exert protective role in vascular function. Am J Respir Crit Care Med 2008, 178:1148-1155

5. Agouni A, Lagrue-Lak-Hal AH, Ducluzeau PH, Mostefai HA, DraunetBusson C, Leftheriotis G, Heymes C, Martinez MC, Andriantsitohaina R: Endothelial dysfunction caused by circulating microparticles from patients with metabolic syndrome. Am J Pathol 2008, 173:1210-1219

6. Tual-Chalot S, Leonetti D, Andriantsitohaina R, Martinez MC Microvesicles: intercellular vectors of biological messages. Mol Inter 2011, 11:88-94

7. Martinez MC, Tual-Chalot S, Leonetti D, Andriantsitohaina R: Microparticles: targets and tools in cardiovascular disease. Trends Pharmacol Sci 2011, 32:659-665

8. Yun $\mathrm{CH}$, Jung KH, Chu K, Kim SH, Ji KH, Park HK, Kim HC, Lee ST, Lee SK, Roh JK: Increased circulating endothelial microparticles and carotid atherosclerosis in obstructive sleep apnea. J Clin Neurol 2010, 6:89-98

9. Geiser T, Buck F, Meyer BJ, Bassetti C, Haeberli A, Gugger M: In vivo platelet activation is increased during sleep in patients with obstructive sleep apnea syndrome. Respiration 2002, 69:229-234

10. Ayers L, Ferry B, Craig S, Nicoll D, Stradling JR, Kohler M: Circulating cell-derived microparticles in patients with minimally symptomatic obstructive sleep apnoea. Eur Respir J 2009, 33:574-580

11. Jelic S, Lederer DJ, Adams T, Padeletti M, Colombo PC, Factor P, LeJemtel TH: Endothelial repair capacity and apoptosis are inversely related in obstructive sleep apnea. Vasc Health Risk Manag 2009, 5:909-920

12. Priou P, Gagnadoux F, Tesse A, Mastronardi ML, Agouni A, Meslier N, Racineux JL, Martinez MC, Trzepizur W, Andriantsitohaina R: Endothelial dysfunction and circulating microparticles from patients with obstructive sleep apnea. Am J Pathol 2010, 177:974-983

13. Trzepizur W, Priou P, Paris A, Nardi J, Tual-Chalot S, Meslier N, Urban T, Andriantsitohaina R, Martinez MC, Gagnadoux F: Nocturnal release of leukocyte-derived microparticles in males with obstructive sleep apnoea. Eur Respir J 2011, 37:1293-1295

14. Kraiczi H, Hedner J, Peker $Y$, Carlson J: Increased vasoconstrictor sensitivity in obstructive sleep apnea. J Appl Physiol 2000, 89:493498

15. Johns MW: A new method for measuring daytime sleepiness: the Epworth sleepiness scale. Sleep 1991, 14:540-545

16. Pelletier-Fleury N, Meslier N, Gagnadoux F, Person C, Rakotonanahary D, Ouksel H, Fleury B, Racineux JL: Economic arguments for the immediate management of moderate-to-severe obstructive sleep apnoea syndrome. Eur Respir J 2004, 23:53-60

17. Priou P, Hamel JF, Person C, Meslier N, Racineux JL, Urban T, Gagnadoux F: Long-terme outcome of noninvasive positive pressure ventilation for obesity hypoventilation syndrome. Chest 2010, 138: 84-90

18. Kushida CA, Littner MR, Morgenthaler T, Alessi CA, Bailey D, Coleman J Jr, Friedman L, Hirshkowitz M, Kapen S, Kramer M, LeeChiong T, Loube DL, Owens J, Pancer JP, Wise M: Practice parameters for the indications for polysomnography and related procedures: an update for 2005. Sleep 2005, 28:499-521

19. Tual-Chalot S, Guibert C, Muller B, Savineau JP, Andriantsitohaina R, Martinez MC: Circulating microparticles from pulmonary hypertensive 
rats induce endothelial dysfunction. Am J Respir Crit Care Med 2010, 182:261-268

20. Tesse A, Meziani F, David E, Carusio N, Kremer H, Schneider F, Andriantsitohaina R: Microparticles from preeclamptic women induce vascular hyporeactivity in vessels from pregnant mice through an overproduction of NO. Am J Physiol Heart Circ Physiol 2007, 293: $\mathrm{H} 520-\mathrm{H} 525$

21. Agouni A, Mostefai HA, Porro C, Carusio N, Favre J, Richard V, Henrion D, Martinez MC, Andriantsitohaina R: Sonic hedgehog carried by microparticles corrects endothelial injury through nitric oxide release. FASEB J 2007, 21:2735-2741

22. Forest $\mathrm{A}$, Pautas $\mathrm{E}$, Ray $\mathrm{P}$, Bonnet $\mathrm{D}$, Verny M, Amabile N, Boulanger C, Riou B, Tedgui A, MallatZ, Boddaert J: Circulating microparticles and procoagulant activity in elderly patients. J Gerontol A Biol Sci Med Sci 2010, 65:414-420

23. Emmerechts J, Loyen S, Hoylaerts MF: Microparticle number or procoagulant activity are not upregulated in healthy elderly persons. Thromb Res 2012, 129:98-100

24. Soriano AO, Wenche J, Chirinos JA, Valdivia MA, Velasquez HS, Jimenez JJ, Horstman LL, Kett DH, Schein RM, Ahn YS: Levels of endothelial and platelet microparticles and their interactions with leukocytes negatively correlate with organ dysfunction and predict mortality in severe sepsis. Crit Care Med 2005, 33:2540-2546
25. Allahdadi KJ, Walker BR, Kanagy NL: Augmented endothelin vasoconstriction in intermittent hypoxia-induced hypertension. Hypertension 2005, 45:705-709

26. Lefevre B, Godin-Ribuot D, Joyeux-Faure M, Caron F, Bessard G, Lévy P, Stanke-Labesque F: Functional assessment of vascular reactivity after chronic intermittent hypoxia in the rat. Respir Physiol Neurobiol 2006, 150:278-286

27. Dematteis M, Julien C, Guillermet C, Sturm N, Lantuejoul S, Mallaret M, Lévy P, Gozal E: Intermittent hypoxia induces early functional cardiovascular remodeling in mice. Am J Respir Crit Care Med 2008 , $177: 227-235$

28. Noda A, Nakata S, Koike Y, Miyata S, Kitaichi K, Nishizawa T, Nagata K, Yasuma F, Murohara T, Yokota M: Continuous positive airway pressure improves daytime baroreflex sensitivity and nitric oxide production in patients with moderate to severe obstructive sleep apnea syndrome. Hypertens Res 2007, 30:669-676

29. Tahawi Z, Orolinova N, Joshua IG, Bader M, Fletcher EC: Altered vascular reactivity in arterioles of chronic intermittent hypoxic rats. J Appl Physiol 2001, 90:2007-2013

30. Jelic S, Padeletti M, Kawut SM, Higgins C, Canfield SM, Onat D, Colombo PC, Basner RC, Factor P, LeJemtel TH: Inflammation, oxidative stress, and repair capacity of the vascular endothelium in obstructive sleep apnea. Circulation 2008, 117:2270-2278 